PROCEEDINGS OF THE AMERICAN MATHEMATICAL SOCIETY

Volume 124, Number 9, September 1996

\title{
THE LOCAL COHOMOLOGY MODULES OF MATLIS REFLEXIVE MODULES ARE ALMOST COFINITE
}

\author{
RICHARD BELSHOFF, SUSAN PALMER SLATTERY, AND CAMERON WICKHAM
}

(Communicated by Wolmer V. Vasconcelos)

\begin{abstract}
We show that if $M$ and $N$ are Matlis reflexive modules over a complete Gorenstein local domain $R$ and $I$ is an ideal of $R$ such that the dimension of $R / I$ is one, then the modules $\operatorname{Ext}_{R}^{i}\left(N, \mathrm{H}_{I}^{j}(M)\right)$ are Matlis reflexive for all $i$ and $j$ if $\operatorname{Supp}(N) \subseteq V(I)$. It follows that the Bass numbers of $\mathrm{H}_{I}^{j}(M)$ are finite. If $R$ is not a domain, then the same results hold for $M=R$.
\end{abstract}

\section{INTRODUCTION}

Let $R$ be a Noetherian local ring with maximal ideal $\mathfrak{m}$ and residue field $k$. If $M$ is a finitely generated module, then the local cohomology modules $\mathrm{H}_{\mathfrak{m}}^{j}(M)$ are artinian, so $\operatorname{Ext}_{R}^{i}\left(k, \mathrm{H}_{\mathfrak{m}}^{j}(M)\right)$ is finitely generated for every $i$ and $j$. Grothendieck $[\mathrm{G}]$ conjectured the following:

For any ideal $I$ and any finitely generated module $M$, the module $\operatorname{Hom}_{R}\left(R / I, \mathrm{H}_{I}^{j}(M)\right)$ is finitely generated for all $j$.

Here, $\mathrm{H}_{I}^{j}(M)$ denotes the $j^{\text {th }}$ local cohomology module of $M$ with support in I. Hartshorne [H2] showed that this is false in general. However, he defined an $R$ module $M$ to be $I$-cofinite if $\operatorname{Supp}(M) \subseteq V(I)$ and $\operatorname{Ext}_{R}^{i}(R / I, M)$ is finitely generated for all $i$ and asked:

For which rings $R$ and ideals $I$ are the modules $\mathrm{H}_{I}^{j}(M) I$-cofinite for all $j$, and all finitely generated modules $M$ ?

Hartshorne answered this question for the following two cases:

(1) If $R$ is a complete regular local ring and $I$ is a nonzero principal ideal, and

(2) If $R$ is a complete regular local ring and $I$ is a prime ideal with $\operatorname{dim} R / I=1$.

Hartshorne's work provides motivation for some more recent results on this question. Huneke and Koh extended Hartshorne's second case. Theorem 4.1 of $[\mathrm{H}-\mathrm{K}]$ shows that if $R$ is a complete Gorenstein domain, $I$ is an ideal of $R$ with $\operatorname{dim} R / I=$ 1 , and $N$ is finitely generated with support in $V(I)$, then $\operatorname{Ext}_{R}^{i}\left(N, \mathrm{H}_{I}^{j}(M)\right)$ is finitely generated for all $i$ and $j$ and for all finitely generated modules $M$.

This result was further extended in $[D]$, Theorem 3. Delfino was able to remove the Gorenstein condition on $R$ as long as $R$ satisfied one of the three conditions (here $K$ denotes a coefficient ring of $R, q$ the uniformizing parameter of $K$ ):

Received by the editors October 24, 1994 and, in revised form, March 22, 1995

1991 Mathematics Subject Classification. Primary 13D45, 13C99, $13 \mathrm{C} 05$.

Key words and phrases. Matlis reflexive module, local cohomology module, Gorenstein ring, Bass number. 
(i) $K$ is a field;

(ii) $K$ is not a field and $q \in I$;

(iii) $K$ is not a field and $q$ is not an element of any minimal prime of $R / I$.

Delfino and Marley have eliminated the complete domain hypothesis entirely. They prove in $[\mathrm{D}-\mathrm{M}]$ that if $M$ is a finitely generated module over a commutative Noetherian $\operatorname{ring} R$ and $I$ is an ideal such that $\operatorname{dim} R / I=1$, then the local cohomology modules $H_{I}^{j}(M)$ are $I$-cofinite for all $j$.

Hartshorne's work also provided motivation for study in the special case when $M=R$. Theorem 2.3 of [H-K] shows that if $R$ is a regular local ring of characteristic $p>0, I$ is any ideal of $R, j>\operatorname{bight}(I)$, and $\operatorname{Hom}\left(R / I, \mathrm{H}_{I}^{j}(R)\right)$ is finitely generated, then $\mathrm{H}_{I}^{j}(R)=0$. Here, $\operatorname{bight}(I)=\max \{\mathrm{ht} \mathfrak{p} \mid \mathfrak{p}$ is a minimal prime ideal of $I\}$.

Huneke and Sharp obtained more information on the structure of $\mathrm{H}_{I}^{j}(R)$ under the same conditions on $R$ and $I$. Theorem 2.1 of [H-S] shows that the Bass numbers of $\mathrm{H}_{I}^{j}(R)$ are finite for all $j$. As a corollary, they show that $\operatorname{Ass}\left(\mathrm{H}_{I}^{j}(R)\right)$ is finite for all $j$. Using the theory of $D$-modules, Lyubeznik [L] obtained analagous results for equicharacteristic rings.

The goal of the present paper is to obtain similar results as above, but for a larger class of modules. We first focus our attention to the case when $R$ is a complete Gorenstein domain and $I$ is an ideal of $R$ such that $\operatorname{dim} R / I=1$. Let $E=E_{R}(k)$ be the injective hull of the residue field and let $(-)^{\vee}$ denote the functor $\operatorname{Hom}(-, E)$. Recall that a module is Matlis reflexive if $M \cong\left(M^{\vee}\right)^{\vee}$. The class of Matlis reflexive modules over a complete ring includes all finitely generated and artinian modules. We show that if $M$ and $N$ are Matlis reflexive with $\operatorname{Supp}(N) \subseteq V(I)$, then $\operatorname{Ext}_{R}^{i}\left(N, \mathrm{H}_{I}^{j}(M)\right)$ is Matlis reflexive for all $i$ and $j$. In fact, $\operatorname{Ext}_{R}^{i}\left(N, \mathrm{H}_{I}^{j}(M)\right)$ is finitely generated for all $i$ when $j>0$. We give an example to show that $\operatorname{Ext}_{R}^{i}\left(N, \mathrm{H}_{I}^{0}(M)\right)$ need not be finitely generated. However, it turns out that this is enough to show that all the Bass numbers of $\mathrm{H}_{I}^{j}(M)$ are finite.

We then remove the domain assumption on $R$ and study the case when $M=R$ and $N$ is finitely generated. In this situation we show that $\operatorname{Ext}_{R}^{i}\left(N, \mathrm{H}_{I}^{j}(R)\right)$ is Matlis reflexive. As a corollary, we show that $\mathrm{H}_{I}^{j}(R)$ has finite Bass numbers for every $j$.

\section{THE DOMAIN CASE}

Throughout the rest of the paper, $R$ will denote a Noetherian local ring with maximal ideal $\mathfrak{m}$ and residue field $k, I$ will denote an ideal of $R$ such that $\operatorname{dim} R / I=$ 1, $E$ will denote the injective hull of the residue field, and $(-)^{\vee}$ will denote the functor $\operatorname{Hom}_{R}(-, E)$. For basic facts about Matlis reflexive modules, we refer the reader to section 3.2 of [St]. In particular, we make extensive use of the following fact:

If $R$ is complete, then an $R$-module $M$ is Matlis reflexive if and only if there is a short exact sequence

$$
0 \rightarrow S \rightarrow M \rightarrow A \rightarrow 0
$$

with $S$ finitely generated and $A$ artinian (see, for example, [E], Proposition 1.3, or [St], Theorem 3.4.13).

Lemma 1. If $M$ and $N$ are Matlis reflexive, then $\operatorname{Ext}_{R}^{i}\left(N, \mathrm{H}_{I}^{0}(M)\right)$ is Matlis reflexive for all $i$. 
Proof. Since $\mathrm{H}_{I}^{0}(M) \subseteq M$, this follows from Theorem 3 of $[\mathrm{Be}]$.

Theorem 2. If $R$ is a complete Gorenstein domain, $M$ is Matlis reflexive, and $N$ is finitely generated with $\operatorname{Supp}(N) \subseteq V(I)$, then $\operatorname{Ext}_{R}^{i}\left(N, \mathrm{H}_{I}^{j}(M)\right)$ is Matlis reflexive. In fact, $\operatorname{Ext}_{R}^{i}\left(N, \mathrm{H}_{I}^{j}(M)\right)$ is finitely generated for all $i$ when $j \geq 1$.

Proof. Since $M$ is Matlis reflexive, there is a short exact sequence

$$
0 \rightarrow S \rightarrow M \rightarrow A \rightarrow 0
$$

with $S$ finitely generated and $A$ artinian. Since $A$ is artinian, we obtain from the induced long exact sequence of local cohomology modules the exact sequence

$$
0 \rightarrow \mathrm{H}_{I}^{0}(S) \rightarrow \mathrm{H}_{I}^{0}(M) \rightarrow A \rightarrow \mathrm{H}_{I}^{1}(S) \rightarrow \mathrm{H}_{I}^{1}(M) \rightarrow 0
$$

and

$$
\mathrm{H}_{I}^{j}(M) \cong \mathrm{H}_{I}^{j}(S)
$$

for $j \geq 2$.

Let $K$ denote the kernel of the map $\mathrm{H}_{I}^{1}(S) \rightarrow \mathrm{H}_{I}^{1}(M)$. Then $K$ is artinian, since $A$ is, so $K$ is Matlis reflexive. Thus, from the short exact sequence

$$
0 \rightarrow K \rightarrow \mathrm{H}_{I}^{1}(S) \rightarrow \mathrm{H}_{I}^{1}(M) \rightarrow 0
$$

we obtain the long exact sequence

$$
\cdots \rightarrow \operatorname{Ext}_{R}^{i}\left(N, \mathrm{H}_{I}^{1}(S)\right) \rightarrow \operatorname{Ext}_{R}^{i}\left(N, \mathrm{H}_{I}^{1}(M)\right) \rightarrow \operatorname{Ext}_{R}^{i+1}(N, K) \rightarrow \cdots .
$$

By Theorem 4.1 of $[\mathrm{H}-\mathrm{K}], \operatorname{Ext}_{R}^{i}\left(N, \mathrm{H}_{I}^{1}(S)\right)$ is finitely generated, hence $\operatorname{Ext}_{R}^{i}\left(N, \mathrm{H}_{I}^{1}(M)\right)$ is finitely generated if and only if $\operatorname{Ext}_{R}^{i+1}(N, K)$ is finitely generated.

Since $K$ is artinian, $\operatorname{Hom}(N, K)$ is also artinian, and since $\operatorname{Hom}(N, K) \subseteq$ $\operatorname{Hom}\left(N, \mathrm{H}_{I}^{1}(S)\right), \operatorname{Hom}(N, K)$ has finite length. So $N \otimes K^{\vee} \cong \operatorname{Hom}(N, K)^{\vee}$ has finite length and hence $\operatorname{Tor}_{i}\left(N, K^{\vee}\right)$ has finite length. Thus $\operatorname{Ext}^{i}(N, K) \cong \operatorname{Tor}_{i}\left(N, K^{\vee}\right)^{\vee}$ has finite length for every $i$. Therefore $\operatorname{Ext}_{R}^{i}\left(N, \mathrm{H}_{I}^{1}(M)\right)$ is finitely generated, and hence Matlis reflexive, for all $i$.

By Theorem 1, $\operatorname{Ext}_{R}^{i}\left(N, \mathrm{H}_{I}^{0}(M)\right)$ is Matlis reflexive. For $j \geq 2$,

$$
\operatorname{Ext}_{R}^{i}\left(N, \mathrm{H}_{I}^{j}(M)\right) \cong \operatorname{Ext}_{R}^{i}\left(N, \mathrm{H}_{I}^{j}(S)\right)
$$

for all $i$, and so by Theorem 4.1 of $[\mathrm{H}-\mathrm{K}], \operatorname{Ext}_{R}^{i}\left(N, \mathrm{H}_{I}^{j}(M)\right)$ is finitely generated, hence Matlis reflexive.

We note that $\operatorname{Ext}_{R}^{i}\left(N, \mathrm{H}_{I}^{0}(M)\right)$ need not be finitely generated. For example, let $N=R / I, M=E$, and $i=0$. Then $\operatorname{Ext}_{R}^{i}\left(N, \mathrm{H}_{I}^{0}(M)\right)=(R / I)^{\vee}$. Since $\operatorname{dim} R / I=1,(R / I)^{\vee}$ is not finitely generated. However, the following corollary shows that this is not too bad.

Recall that the $i^{t h}$ term $E^{i}(M)$ in the minimal injective resolution of an $R$ module $M$ is uniquely determined, up to isomorphism, by $M$. In fact, there is a family $\mathcal{F}$ of prime ideals of $R$ such that $E^{i}(M) \cong \bigoplus_{\mathfrak{q} \in \mathcal{F}} E(R / \mathfrak{q})$. The $i^{\text {th }}$ Bass number with respect to $\mathfrak{p}$, denoted $\mu_{i}(\mathfrak{p}, M)$, of $M$ is defined to be the number of copies of the injective hull of $R / \mathfrak{p}$ in this direct sum decomposition of $E^{i}(M)$. By 2.7 of [B], this is the same as the vector space dimension of $\left(\operatorname{Ext}_{R}^{i}(R / \mathfrak{p}, M)\right)_{\mathfrak{p}}$ over $(R / \mathfrak{p})_{\mathfrak{p}}$. We refer to the numbers $\mu_{i}(\mathfrak{p}, M)$ for all $i$ and all $\mathfrak{p}$ collectively as the Bass numbers of $M$. 
Corollary 3. If $R$ is a complete Gorenstein domain and $M$ is a Matlis reflexive $R$-module, then the Bass numbers of $\mathrm{H}_{I}^{j}(M)$ are finite for every $j$.

Proof. Let $k$ be the residue field of $R$. Then $\operatorname{Ext}_{R}^{i}\left(k, \mathrm{H}_{I}^{j}(M)\right)$ is Matlis reflexive by Theorem 2. Since $\operatorname{Ext}_{R}^{i}\left(k, \mathrm{H}_{I}^{j}(M)\right)$ is also a $k$ vector space, it must be finitely generated. If $\mathfrak{p}$ is any nonmaximal prime, it follows from Proposition 1.3 of $[\mathrm{E}]$ that $M_{\mathfrak{p}}$ is finitely generated over $R_{\mathfrak{p}}$. Since $\operatorname{dim} R / I=1$, we have $\left(\mathrm{H}_{I}^{j}(M)\right)_{\mathfrak{p}} \cong$ $\mathrm{H}_{\mathfrak{p} R_{\mathfrak{p}}}^{j}\left(M_{\mathfrak{p}}\right)$ if $\mathfrak{p} \supseteq I$ or $\left(\mathrm{H}_{I}^{j}(M)\right)_{\mathfrak{p}} \cong 0$ if $\mathfrak{p} \nsupseteq I$. In either case, it follows that $\left(\operatorname{Ext}_{R}^{i}\left(R / \mathfrak{p}, \mathrm{H}_{I}^{j}(M)\right)\right)_{\mathfrak{p}}$ is finitely generated over $R_{\mathfrak{p}}$.

Lemma 4. Let $R$ be a complete Gorenstein domain, $M$ a Matlis reflexive module, and $N$ an artinian module. Then $\operatorname{Ext}_{R}^{i}\left(N, \mathrm{H}_{I}^{j}(M)\right)$ is finitely generated (and thus Matlis reflexive) for all $i$ and $j$.

Proof. Since $M$ is Matlis reflexive, $\mathrm{H}_{I}^{j}(M)$ has finite Bass numbers by Corollary 3. Fix $j$. Let $0 \rightarrow \mathrm{H}_{I}^{j}(M) \rightarrow \mathbf{J}^{*}$ be a minimal injective resolution of $\mathrm{H}_{I}^{j}(M)$. Then for each $t$,

$$
\operatorname{Hom}\left(N, J^{t}\right) \cong \bigoplus \operatorname{Hom}(N, E)
$$

since $N$ is artinian. Since $\mathrm{H}_{I}^{j}(M)$ has finite Bass numbers, the direct sum is a finite direct sum. By Matlis duality, $\operatorname{Hom}(N, E)$ is finitely generated. Thus $\operatorname{Hom}\left(N, J^{t}\right)$, and hence $\operatorname{Ext}_{R}^{i}\left(N, \mathrm{H}_{I}^{j}(M)\right)$, is finitely generated.

Theorem 5. If $R$ is a complete Gorenstein domain and $M$ and $N$ are Matlis reflexive such that $\operatorname{Supp}(N) \subseteq V(I)$, then $\operatorname{Ext}_{R}^{i}\left(N, \mathrm{H}_{I}^{j}(M)\right)$ is Matlis reflexive for all $i$ and $j$. In fact, $\operatorname{Ext}_{R}^{i}\left(N, \mathrm{H}_{I}^{j}(M)\right)$ is finitely generated for all $i$ when $j>0$.

Proof. For $j=0$, this is just Lemma 1. Fix $j>0$. Since $N$ is Matlis reflexive, there is a short exact sequence

$$
0 \rightarrow T \rightarrow N \rightarrow B \rightarrow 0
$$

with $T$ finitely generated and $B$ artinian. This induces a long exact sequence

$$
\cdots \rightarrow \operatorname{Ext}_{R}^{i}\left(B, \mathrm{H}_{I}^{j}(M)\right) \rightarrow \operatorname{Ext}_{R}^{i}\left(N, \mathrm{H}_{I}^{j}(M)\right) \rightarrow \operatorname{Ext}_{R}^{i}\left(T, \mathrm{H}_{I}^{j}(M)\right) \rightarrow \cdots .
$$

By Theorem 2, $\operatorname{Ext}_{R}^{i}\left(T, \mathrm{H}_{I}^{j}(M)\right)$ is finitely generated. By Lemma $4, \operatorname{Ext}_{R}^{i}\left(B, \mathrm{H}_{I}^{j}(M)\right)$ is finitely generated. Thus $\operatorname{Ext}_{R}^{i}\left(N, \mathrm{H}_{I}^{j}(M)\right)$ is finitely generated, and hence Matlis reflexive for all $i$ when $j>0$.

\section{THE NONDOMAIN CASE}

In this section we drop the domain requirement on the $\operatorname{ring} R$ and obtain similar results for the local cohomology modules of $R$. As one of our main tools, we use Grothendieck's local duality theorem. In the special case when $R$ is a Gorenstein ring with $\operatorname{dim} R=d$, local duality states that, for any finitely generated $R$-module $M$,

$$
\mathrm{H}_{\mathfrak{m}}^{i}(M) \cong \operatorname{Ext}_{R}^{d-i}(M, R)^{\vee}
$$

for every $i$. For details the reader is referred to [H1].

Lemma 6. If $R$ is a complete Gorenstein ring and $N$ is finitely generated with $\operatorname{Supp}(N) \subseteq V(I)$, then $\operatorname{Ext}_{R}^{i}\left(N, \mathrm{H}_{I}^{j}(R)\right)$ is Matlis reflexive for all $i$ and $j$. 
Proof. By Lemma 2 of [D], we may assume that $N=R / I$. Let $0 \rightarrow R \rightarrow \mathbf{D}^{*}$ be an injective resolution of $R$. Since $R$ is Gorenstein, $D^{t}=(\bigoplus E(R / \mathfrak{p}) ;$ ht $\mathfrak{p}=t)$, so $\mathrm{H}_{I}^{0}\left(\mathbf{D}^{*}\right)$ is the complex

$$
0 \rightarrow E^{\prime} \stackrel{\psi}{\rightarrow} E \rightarrow 0
$$

where $E^{\prime}=(\bigoplus E(R / \mathfrak{p})$; ht $\mathfrak{p}=d-1, \mathfrak{p} \supseteq I), E$ is the injective hull of the residue field, and $d=\operatorname{dim} R$. Thus $\mathrm{H}_{I}^{j}(R)=0$ for $j \neq d-1, d$, and there is a short exact sequence

$$
0 \rightarrow \mathrm{H}_{I}^{d-1}(R) \rightarrow E^{\prime} \rightarrow A \rightarrow 0
$$

where $A$ is the image of $\psi$. It follows that there is an exact sequence

$$
\begin{aligned}
0 & \rightarrow \operatorname{Hom}\left(R / I, \mathrm{H}_{I}^{d-1}(R)\right) \rightarrow \operatorname{Hom}\left(R / I, E^{\prime}\right) \\
& \rightarrow \operatorname{Hom}(R / I, A) \rightarrow \operatorname{Ext}^{1}\left(R / I, \mathrm{H}_{I}^{d-1}(R)\right) \rightarrow 0
\end{aligned}
$$

and that $\operatorname{Ext}^{i}\left(R / I, \mathrm{H}_{I}^{d-1}(R)\right) \cong \operatorname{Ext}^{i-1}(R / I, A)$ for $i>1$. Since $A$ is artinian, it follows that $\operatorname{Ext}^{i}\left(R / I, \mathrm{H}_{I}^{d-1}(R)\right)$ is artinian, and hence Matlis reflexive, for $i>1$.

Consider the complex

$$
0 \rightarrow \operatorname{Hom}\left(R / I, E^{\prime}\right) \stackrel{\operatorname{Hom}(R / I, \psi)}{\longrightarrow} \operatorname{Hom}(R / I, E) \rightarrow 0 .
$$

Let $J$ denote the image of $\operatorname{Hom}(R / I, \psi)$ and $K$ denote the kernel of $\operatorname{Hom}(R / I, \psi)$. Then $K=\operatorname{Ext}_{R}^{d-1}(R / I, R)$ and $\operatorname{Hom}(R / I, E) / J=\operatorname{Ext}_{R}^{d}(R / I, R)$. Using the left exactness of $\operatorname{Hom}(R / I,-)$ and local duality, we have

$$
\operatorname{Hom}\left(R / I, \mathrm{H}_{I}^{d-1}(R)\right) \cong K \cong\left(\mathrm{H}_{\mathfrak{m}}^{1}(R / I)\right)^{\vee}
$$

and

$$
\operatorname{Ext}^{1}\left(R / I, \mathrm{H}_{I}^{d-1}(R)\right) \cong \operatorname{Hom}(R / I, A) / J \subseteq \operatorname{Hom}(R / I, E) / J \cong\left(\mathrm{H}_{\mathfrak{m}}^{0}(R / I)\right)^{\vee} .
$$

Since $R$ is complete and $\mathrm{H}_{\mathfrak{m}}^{s}(R / I)$ is artinian for any $s$, we have $\operatorname{Ext}^{i}\left(R / I, \mathrm{H}_{I}^{d-1}(R)\right)$ is finitely generated for $i=0,1$, and hence Matlis reflexive.

Finally, note that $\mathrm{H}_{I}^{d}(R)$ is a factor of $E$, hence artinian. Therefore, $\operatorname{Ext}^{i}\left(R / I, \mathrm{H}_{I}^{d}(R)\right)$ is artinian, and hence Matlis reflexive, for all $i$.

Corollary 7. If $R$ is a complete Gorenstein ring, then $\mathrm{H}_{I}^{j}(R)$ has finite Bass numbers for every $j$.

Proof. By Lemma 6, $\operatorname{Ext}_{R}^{i}\left(k, \mathrm{H}_{I}^{j}(R)\right)$ is Matlis reflexive. The proof then proceeds as in Corollary 3.

Lemma 8. If $R$ is a complete Gorenstein ring and $N$ is an artinian module, then the module $\operatorname{Ext}_{R}^{i}\left(N, \mathrm{H}_{I}^{j}(R)\right)$ is finitely generated for all $i$ and $j$.

Proof. Since $\mathrm{H}_{I}^{j}(R)$ has finite Bass numbers, the proof of Lemma 4 may be adapted.

Theorem 9. If $R$ is a complete Gorenstein ring and $N$ is a Matlis reflexive module with $\operatorname{Supp}(N) \subseteq V(I)$, then $\operatorname{Ext}_{R}^{i}\left(N, \mathrm{H}_{I}^{j}(R)\right)$ is Matlis reflexive for all $i$ and $j$.

Proof. Since $N$ is Matlis reflexive, there is a short exact sequence

$$
0 \rightarrow T \rightarrow N \rightarrow B \rightarrow 0
$$

with $T$ finitely generated and $B$ artinian. This induces a long exact sequence

$$
\cdots \rightarrow \operatorname{Ext}_{R}^{i}\left(B, \mathrm{H}_{I}^{j}(R)\right) \rightarrow \operatorname{Ext}_{R}^{i}\left(N, \mathrm{H}_{I}^{j}(R)\right) \rightarrow \operatorname{Ext}_{R}^{i}\left(T, \mathrm{H}_{I}^{j}(R)\right) \rightarrow \cdots .
$$


By Lemma 6, $\operatorname{Ext}_{R}^{i}\left(T, \mathrm{H}_{I}^{j}(R)\right)$ is Matlis reflexive for all $j$. By Lemma 8, $\operatorname{Ext}_{R}^{i}\left(B, \mathrm{H}_{I}^{j}(R)\right)$ is finitely generated for all $j$. Thus $\operatorname{Ext}_{R}^{i}\left(N, \mathrm{H}_{I}^{j}(R)\right)$ is Matlis reflexive for all $i$ and $j$.

\section{REFERENCES}

[B] H. Bass, On the ubiquity of Gorenstein rings, Math. Z. 82 (1963), 8-28. MR 27:3669

[Be] R. Belshoff, Some change of ring theorems for Matlis reflexive modules, Comm. Algebra 22 (1994), 3545-3552. CMP 94:13

[D] D. Delfino, On the cofiniteness of local cohomology modules, Math. Proc. Camb. Phil. Soc. 115 (1994), 79-84. MR 94m:13023

[D-M] D. Delfino and T. Marley, Cofinite Modules and Local Cohomology, preprint.

[E] E. Enochs, Flat covers and flat cotorsion modules, Proc. Amer. Math. Soc. (2) 92 (1984), 179-184. MR 85j: 13016

[G] A. Grothendieck, Cohomologie locale des faisceaux cohérents et théorèmes de Lefshcetz locaux et globaux. S.G.A. II. North Holland, 1968. MR 57:16294

[H1] R. Hartshorne, "Residues and Duality," Lecture Notes in Mathematics, No. 20, SpringerVerlag, 1966. MR 36:5145

[H2] R. Hartshorne, Affine duality and cofiniteness, Invent. Math. 9 (1970), 145-164. MR 41:1750

[H-K] C. Huneke and J. Koh, Cofiniteness and vanishing of local cohomology modules, Math. Proc. Camb. Phil. Soc. 110 (1991), 421-429. MR 92g:13021

[H-S] C. Huneke and R. Sharp, Bass numbers of local cohomology modules, Trans. Amer. Math. Soc. 339 (1993), 765-779. MR 93m:13008

[L] G. Lyubeznik, Finiteness properties of local cohomology modules, Invent. Math. 113 (1993), 41-55. MR 94e:13032

[St] J. R. Strooker, Homological Questions in Local Algebra, London Mathematical Society Lecture Notes 145, Cambridge University Press, Cambridge, 1990. MR 91m:13013

Department of Mathematics, Southwest Missouri State University, Springfield, MisSOURI 65804

E-mail address: rgb865f@cnas.smsu.edu

Current address: S. P. Slattery: Department of Mathematics, Alabama State University, Montgomery, Alabama 36101

E-mail address: slattery@asu.alasu.edu

E-mail address: cgw121f@cnas.smsu.edu 